\title{
Aufbau der ISO 50001
}

Der neue Aufbau der ISO 50001 stellt ein hohes Maß an Komptabilität mit anderen Normen sicher. Der bisherige an einen Deming-Kreis angegliederte Aufbau der Norm in die Schritte Planung (Plan), Umsetzung (Do), Prüfung (Check) und Korrekturen (Act) wurde beibehalten. Die Kap. 1 bis 4 enthalten allgemeine Grundlagen, der klassische PDCA-Zyklus wird über die Kap. 5 bis 11 abgebildet. 